\title{
Evaluación de la Calidad de Aire en el Proyecto de Explotación Cantera Camucha, distrito de Morococha, provincia de Yauli, Junín
}

\author{
Air Quality Assessment in the Camucha Quarry Exploitation Project, Morocco \\ district, Yauli province, Junín
}

Luis Ricardo Licla Tomayro ${ }^{1}$

Recibido: 24/06/2021 - Aprobado: 03/10/2021 - Publicado: 23/12/2021

\begin{abstract}
RESUMEN
La presente investigación tuvo como objetivo evaluar la calidad de aire en el Proyecto de Explotación Cantera Camucha, localizado en el distrito de Morococha, provincia de Yauli, región de Junín. Para ello se utilizó el método gravimétrico establecido en el Protocolo Nacional de Monitoreo de Calidad de Aire (SINIA, 2019) con el propósito de determinar la concentración de material particulado (PM10 y PM2.5) a barlovento y sotavento del área de explotación. Los resultados demuestran que los niveles de material particulado cumplen con los valores máximos permitidos en los Estándares de Calidad Ambiental (ECA) para Aire, lo cual indica que la calidad de aire no constituye un riesgo para la salud de los trabajadores ni al medioambiente.
\end{abstract}

Palabras claves: Calidad de aire; material particulado; Cantera.

\section{ABSTRACT}

The objective of this research was to evaluate the air quality in the Camucha Quarry Exploitation Project, located in the Morococha district, Yauli province, Junín region. For this, the gravimetric method established in the National Protocol for the Monitoring of Environmental Air Quality (MINAM, 2017) was used, for the purpose of determining the concentration of particulate material (PM10 and PM2.5) to windward and leeward of the area of exploitation. The results show that the levels of particulate matter comply with the maximum values allowed in the Environmental Quality Standards (ECA) for Air, which indicates that air quality does not represent a risk to worker's health or the environment.

Keywords: Air quality; particulate matter; Quarry.

\footnotetext{
1 Ingeniero Ambiental de la Universidad Nacional Agraria La Molina. Lima, Perú. Universidad Nacional Mayor de San Marcos, Facultad de Ingeniería Geológica, Minera, Metalúrgica y Geográfica, Lima, Perú. Egresado de la Maestría en Ciencias Ambientales.
}

E-mail: luis.licla@unmsm.edu.pe - ORCID: https://orcid.org/0000-0002-8643-5675 


\section{INTRODUCCIÓN}

La contaminación del aire es un problema global que pone en riesgo la salud y el bienestar de millones de personas (Guzman Vasquez, 2019). De acuerdo con la OMS (2016), la contaminación del aire provoca la muerte de 3 millones de personas al año por problemas respiratorios y cardiovasculares, es por ello, que se posiciona como uno de los mayores problemas de salud ambiental en la actualidad.

Entre las principales fuentes de contaminación destacan las fuentes fijas asociadas a la actividad industrial, comercial, y minera, fuentes móviles que se relaciones con la actividad de transporte motorizado como los automóviles, camiones, buses, aviones, etc., y las fuentes naturales que comprenden incendios forestales, actividad volcánica, erosión entre otros (IDEAM, 2019).

En el Perú la contaminación del aire afecta mayormente las zonas urbanas e industriales, siendo el mayor contribuyente de contaminación por las emisiones de gases y material particulado, el parque automotor (Blanco \& Paricahua, 2020), esto se debe a la presencia de vehículos antiguos, los cuales contaminan y consumen más combustible que uno nuevo (Valverde Flores, 2017).

Por otra parte, La Rotta Latorre \& TorresT ovar (2017) establecen que la actividad minera también genera un porcentaje importante de emisiones de material particulado dependiendo del método de explotación, lo cual puede deteriorar la función pulmonar, provocar infecciones respiratorias, y en casos extremos cáncer pulmonar en las personas expuestas a niveles altos de material particulado $\left(\mathrm{PM}_{10}\right.$ y $\left.\mathrm{PM}_{2.5}\right)$. Por tanto, es necesario evaluar periódicamente si las partículas emitidas en un determinado proyecto exceden los Estándares de Calidad Ambiental (ECA), con el propósito de implementar nuevas medidas de manejo ambiental, que permitan salvaguardar la salud de los trabajadores y proteger el medio ambiente (Díaz Lazo, 2009).

La presente investigación fue elaborada en base a los resultados del monitoreo ambiental de $\mathrm{PM}_{10}$ y $\mathrm{PM}_{2.5}$ realizado por CMI Vilca Hermanos S.A.C. en el periodo 2018-2020, como parte de los compromisos ambientales asumidos en su instrumento de gestión ambiental aprobado.

\section{METODOS}

\section{1. Área de Estudio}

El Proyecto de Explotación Cantera Camucha se ubica en el distrito de Morococha, provincia de Yauli, región de Junín, a $8.5 \mathrm{~km}$ del centro poblado Nueva Morococha (Figura 1). Este proyecto tiene como objetivo realizar las labores de explotación de arcilla en el yacimiento denominado Cantera Camucha, perteneciente a la unidad estratigráfica Depósitos Glaciares (Q-gl), la cual está constituido por depósitos morréicos, y bloques rellenados con arcillas, limos y arenas.

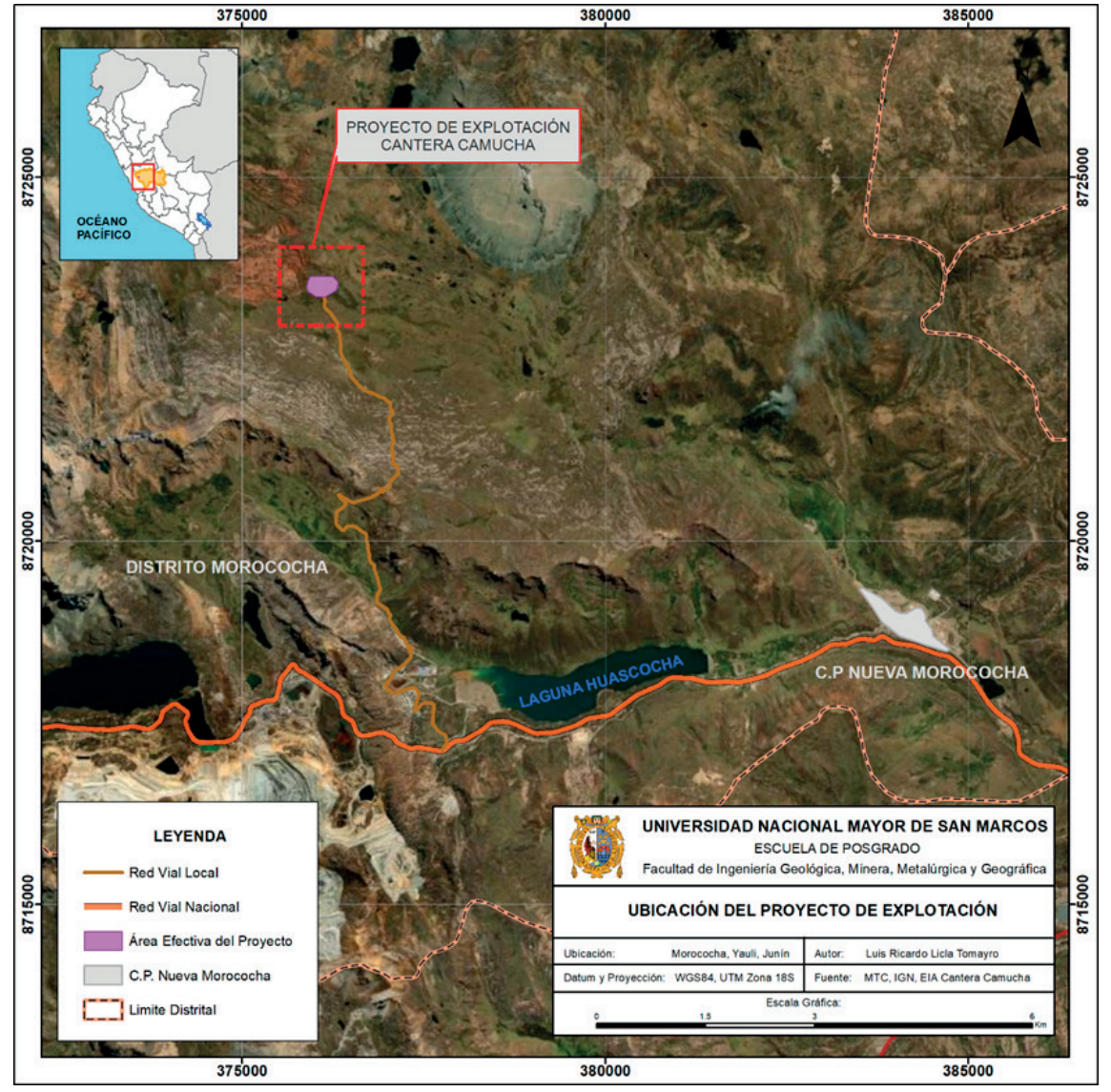

Figura 1. Ubicación del Proyecto de Explotación

Fuente: Imagen de Satelital de Google Earth Pro - Setiembre 2020 
El método de explotación en la Cantera Camucha es de tajo abierto mediante bancos de explotación, y se emplea únicamente una excavadora con cuchara de $1 \mathrm{~m}^{3}$, volquetes mineros, y herramientas manuales como barretas, picos y lampas. La labor minera comprende las etapas de: decapeo, desbroce, extracción, carguío, almacenamiento, y transporte.

\subsection{Metodología de Monitoreo}

Para la determinación $\mathrm{PM}_{10}$ y $\mathrm{PM}_{2.5}$ se empleó el método gravimétrico tal como lo establece el Protocolo Nacional de Monitoreo de Calidad del Aire (SINIA, 2019). Para ello se utilizó un muestreador de partículas de alto volumen (Equipo Hi-Vol), el cual permite la recolección de partículas gruesas con diámetro aerodinámico menor o igual a 10 micrómetros $\left(\mathrm{PM}_{10}\right)$, y menor a 2.5 micrómetros $\left(\mathrm{PM}_{2.5}\right)$, en filtros de cuarzo previamente pesados. Estas muestras son recolectadas durante 24 horas, para luego determinar el peso de cada fracción de partículas $\left(\mathrm{PM}_{10}\right.$ y $\left.\mathrm{PM}_{2.5}\right)$ en un laboratorio acreditado por el Instituto Nacional de Calidad (INACAL).

\subsubsection{Equipos de Monitoreo}

En la Tabla 1, se precisa los equipos de monitoreo ambiental utilizados:
Tabla 1. Equipos de Monitoreo Ambiental

\begin{tabular}{lcc}
\hline Equipo & Marca & Modelo \\
\hline GPS & Garmin & GPSMAP \\
Muestreador de Partículas & Tish Enviromental & Volumétrico \\
Muestreador de Partículas & Tish Enviromental & Volumétrico \\
Estación Meteorológica & Davis Instruments & Vantage Pro 2 \\
\hline
\end{tabular}

Fuente: Elaboración Propia

Es importante señalar que estos equipos de monitoreo contaban con su certificado de calibración vigente emitido por INACAL, en las fechas en donde se realizaron los monitoreos en campo.

\subsubsection{Estaciones de Monitoreo}

De acuerdo con lo establecido en el instrumento de gestión ambiental aprobado del Proyecto de Explotación Cantera Camucha, se han definido dos estaciones de monitoreo de calidad de aire a sotavento y barlovento (Tabla 2, Figura 2), las cuales que se ubican en las siguientes coordenadas UTM.

Tabla 2. Estaciones de Monitoreo

\begin{tabular}{lllccc}
\hline \multirow{2}{*}{ Factor Ambiental } & \multirow{2}{*}{ Estación } & \multirow{2}{*}{ Descripción } & \multicolumn{2}{c}{ Coordenadas UTM WGS84 } & \multicolumn{2}{c}{ Altitud } \\
& & & Norte & Este & m.s.n.m. \\
\hline \multirow{2}{*}{ Calidad de Aire } & CA-1 & A Sotavento del área de explotación & 8723649 & 375909 & 4670 \\
& CA-2 & A Barlovento del área de explotación & 8723284 & 376231 & 4650 \\
\hline
\end{tabular}

Fuente: Elaboración Propia

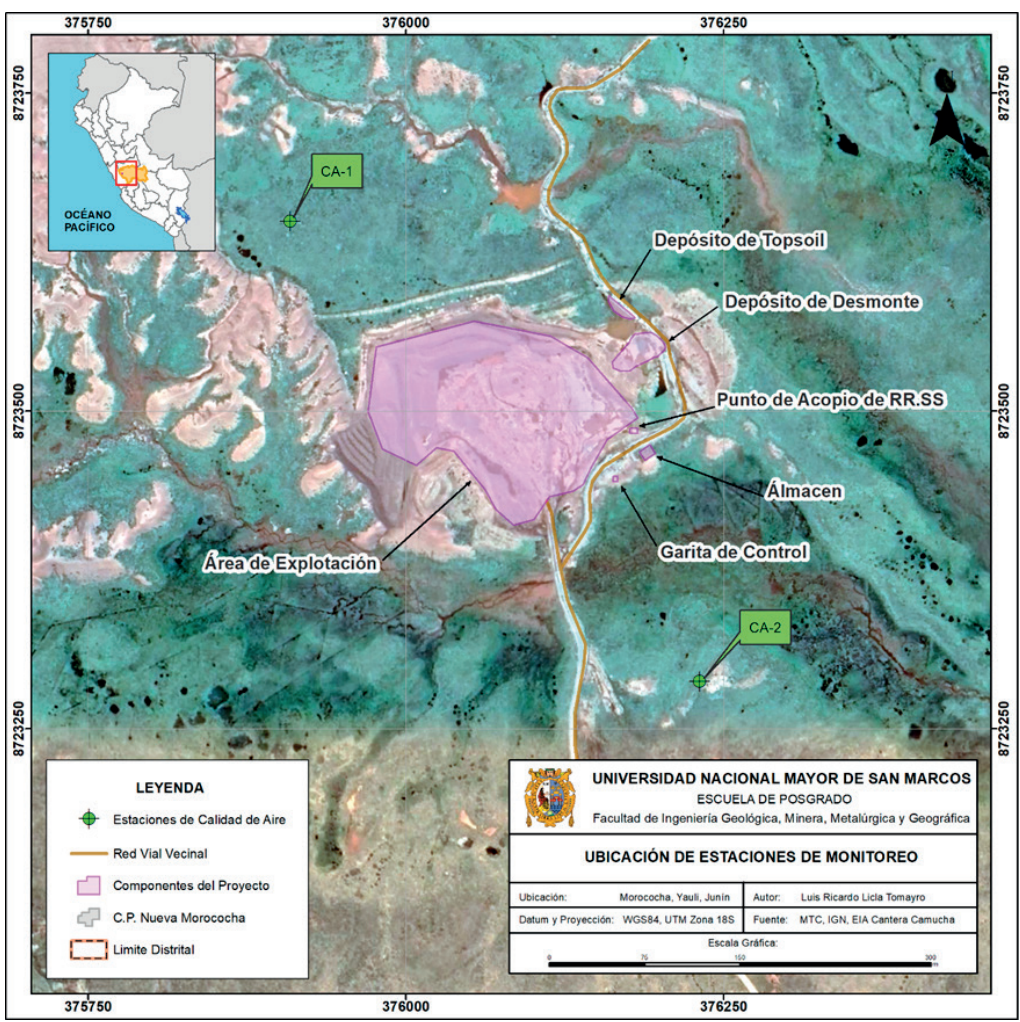

Figura 1. Ubicación del Proyecto de Explotación

Fuente: Imagen de Satelital de Google Earth Pro - Setiembre 2020 


\subsubsection{Estándares de Calidad Ambiental}

Finalmente, en la Tabla 3 se establecen los ECA para Aire vigentes a nivel nacional:

Tabla 3. Valores de Concentración Máxima de Material Particulado

\begin{tabular}{llll}
\hline Contaminante & Periodo & Valor $\left(\boldsymbol{\mu g} / \mathrm{m}^{3}\right)$ & Método de Análisis \\
\hline $\mathrm{PM}_{10}$ & 24 horas & 100 & Gravimetría \\
$\mathrm{PM}_{2.5}$ & 24 horas & 50 & Gravimetría \\
\hline
\end{tabular}

Fuente: MINAM, 2017

\section{RESULTADOS}

En la tabla 4 se presentan los resultados obtenidos del monitoreo ambiental realizado en el periodo 2018-2020.

Los valores han sido comparados con los Estándares de Calidad Ambiental (ECA) vigente a nivel nacional, a fin de determinar su cumplimiento.
Los resultados del monitoreo ambiental presentados en la Figura 3 y Figura 4, demuestran que la concentración de material particulado $\mathrm{PM}_{10}$ y $\mathrm{PM}_{2,5}$ en las estaciones CA-1 y CA-2, se encuentran por debajo de los Estándares de Calidad Ambiental (ECA) para Aire de $100 \mu \mathrm{g} / \mathrm{m}^{3}$ y 50 $\mu \mathrm{g} / \mathrm{m}^{3}$ respectivamente.

Adicionalmente al muestreo de $\mathrm{PM}_{10}$ y $\mathrm{PM}_{2.5}$ se registró la velocidad y dirección del viento en las estaciones CA-1 y CA-2, debido a que estos parámetros son importantes para evaluar la dispersión de contaminantes en el área del proyecto. En la Figura 5 y Figura 6, se detalla las rosas de vientos generadas en las estaciones de monitoreo.

Los resultados indican que el viento presento un mayor porcentaje de ocurrencia para velocidades entre 1.6 y $3.4 \mathrm{~m} / \mathrm{s}$ (Figura 5 y Figura 6), categorizado como brisa suave según la escala de Beaufort, así también, respecto a la

Tabla 4. Resultados del monitoreo de Material Particulado $\mathrm{PM}_{10}$ y $\mathrm{PM}_{2.5}$

\begin{tabular}{lllll}
\hline \multirow{2}{*}{ Parámetro } & Monitoreo & \multicolumn{2}{l}{ Estación de Monitoreo } & ECA AIRE \\
& CA-1 & CA-2 & D.S. N 003-2017-MINAM $^{2.5}$ \\
\hline & Diciembre 2018 & 3.56 & $<3.15$ & \\
$\mathrm{PM}_{10}$ & 10.15 & $<3.15$ & $100 \mu \mathrm{g} / \mathrm{m}^{3}$ \\
$\left(\mu \mathrm{g} / \mathrm{m}^{3}\right)$ & Junio 2019 & 5.58 & $<3.15$ & \\
& Diciembre 2019 & 10.23 & $<3.15$ & \\
& Junio 2020 & 8.50 & $<3.15$ & \\
& Diciembre 2020 & 2.96 & $<2.50$ & $50 \mu \mathrm{g} / \mathrm{m}^{3}$ \\
& Diciembre 2018 & 3.83 & $<2.50$ & \\
$\mathrm{PM}_{25}$ & Junio 2019 & 4.36 & $<2.50$ & \\
\hline & Diciembre 2019 & 4.18 & $<2.50$ & \\
& Junio 2020 & 3.90 & $<2.50$ & \\
\hline
\end{tabular}

Fuente: Elaboración Propia

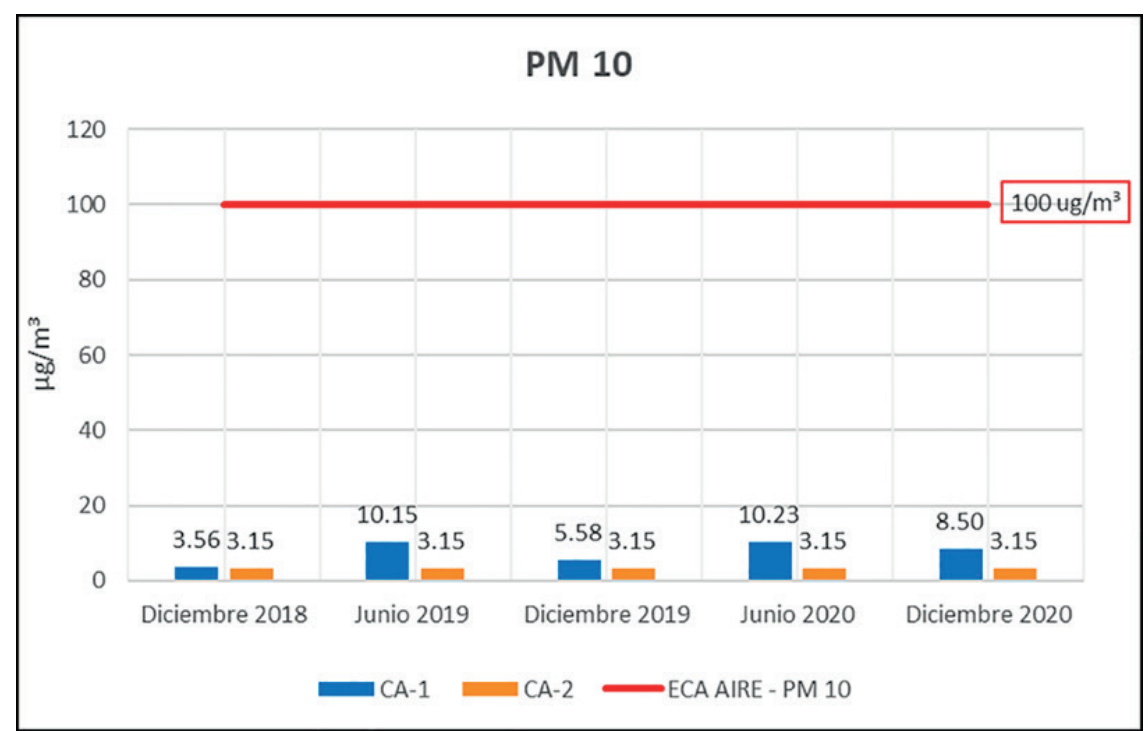

Figura 3. Niveles de concentración de $\mathrm{PM}_{10}$. Descripción: Los valores de $\mathrm{PM}_{10}$ se encuentran expresados en $\mu \mathrm{g} / \mathrm{m}^{3}$ tal como lo establecen Estándares de Calidad Ambiental (ECA) para Aire 


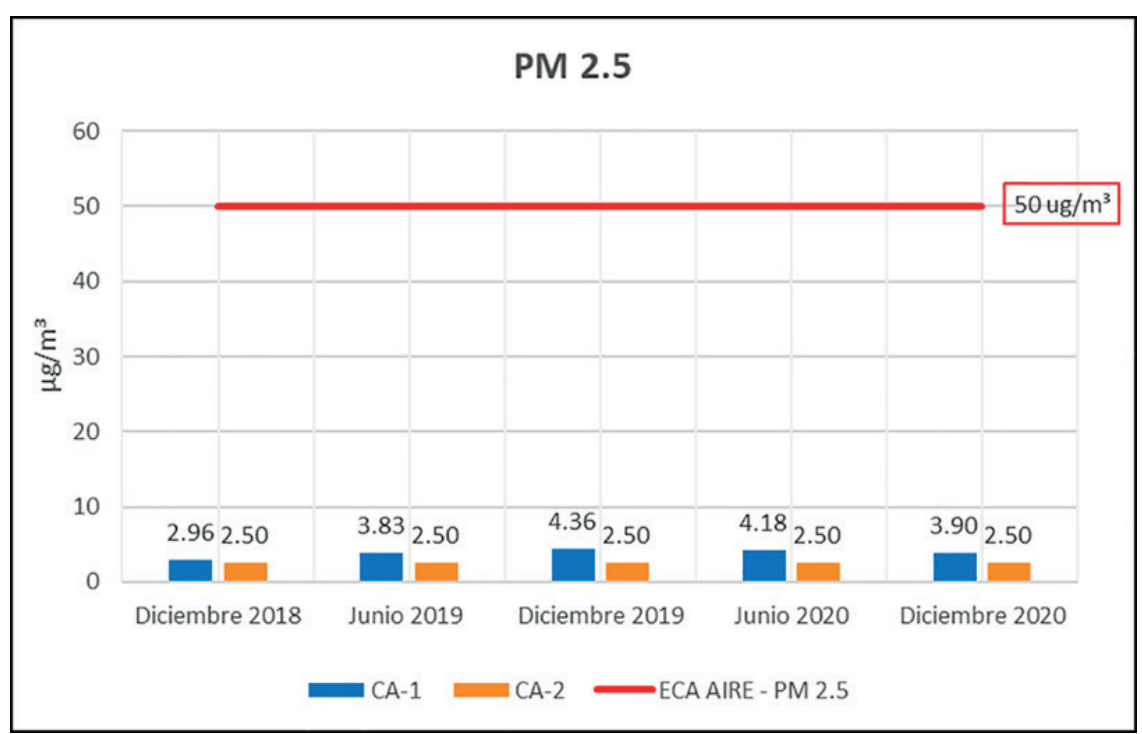

Figura 4. Niveles de concentración de PM2.5. Descripción: Los valores de $\mathrm{PM}_{2.5}$ se encuentran expresados en $\mu \mathrm{g} / \mathrm{m}^{3}$ tal como lo establecen Estándares de Calidad Ambiental (ECA) para Aire

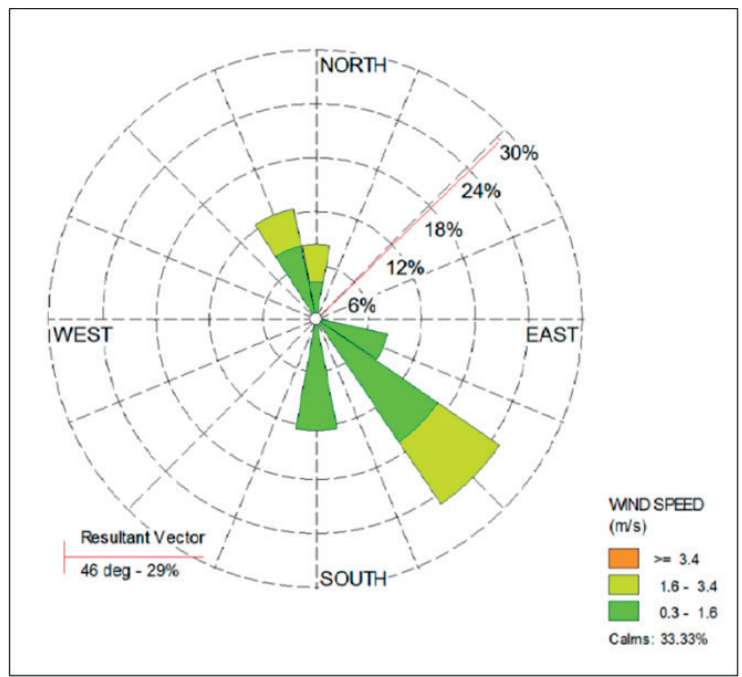

Figura 5. Rosa de Viento en la Estación CA-2 (Sotavento) Fuente: Elaboración Propia en el Software WRPLOT

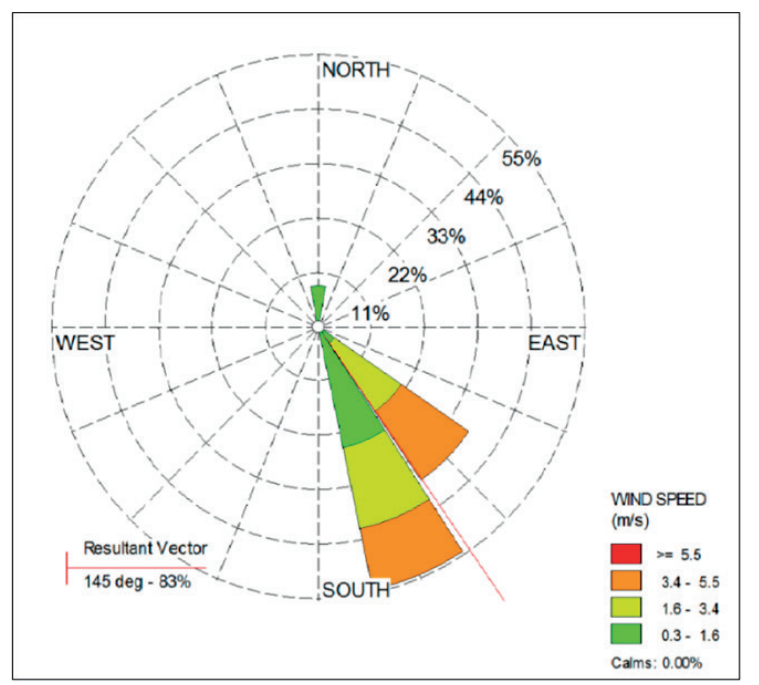

Figura 6. Rosa de Viento en la Estación CA-2 (Barlovento) Fuente: Elaboración Propia en el Software WRPLOT 
dirección del viento, predominaron los vientos provenientes del Sureste (SE) en las dos estaciones de monitoreo.

\section{DISCUSIÓN}

La concentración de material particulado menor a 10 micras $\left(\mathrm{PM}_{10}\right)$ y menor a 2.5 micras $\left(\mathrm{PM}_{2.5}\right)$ en el área efectiva del Proyecto de Explotación Cantera Camucha (Figura 3 y Figura 4) no exceden los Estándares de Calidad Ambiental (ECA) para aire (MINAM, 2017), lo cual indica que los niveles material particulado que se generan en las labores de explotación (decapeo, desbroce, extracción, carguío, almacenamiento y transporte), no representan un riego significativo para la salud de los trabajadores ni para el medio ambiente.

Asimismo, el cumplimiento Estándares de Calidad Ambiental (ECA) para Aire, evidencia que las medidas de prevención, mitigación y control implementadas por el CMI Vilca Hermanos S.A.C. tales como el riego periódico en zonas de trabajo, mantenimiento preventivo de vehículos y maquinarias, mantenimiento de caminos de acceso, entre otros, reducen eficientemente la generación de material particulado en el área efectiva del proyecto. Este resultado coincide con lo establecido por Díaz Lazo (2009), que indica que las medidas manejo ambiental permiten reducir los niveles de material particulado y gases de combustión en áreas de actividad minera.

Por otra parte, en la Tabla 4 se muestra que las concentraciones de $\mathrm{PM}_{10}$ y $\mathrm{PM}_{2.5}$ registradas en la estación CA-1 (sotavento) son mayores que las concentraciones registradas en la estación CA-2 (barlovento), esto se debe a que el material particulado es transportado por los vientos provenientes del Sureste (SE) hacia la superficie de sotavento (Figura 2), incrementando la concentración de material particulado en esta área. Este resultado guarda relación con lo que sostienen Blanco \& Paricahua (2020), quienes señalan que el viento actúa como un agente de transporte y dispersión de contaminantes del aire ambiental (Blanco Obregón, 2018).

Respecto a la población vulnerable (niños, adultos mayores y madres gestantes) que pudiera ser afectada por las emisiones de material particulado, se debe precisar que no existe ningún centro poblado en el área de actividad minera (Figura 1), siendo el más cercano el centro poblado Nueva Morococha ubicado a $8.5 \mathrm{~km}$ del proyecto y en sentido contrario a la dirección predominante del viento, por lo cual se prevé que las emisiones de material particulado no tendrán ningún impacto en la salud de sus habitantes.

\section{CONCLUSIONES}

La concentración de $\mathrm{PM}_{10}$ (partículas menores a $10 \mu \mathrm{m}$ ) registrada en el área efectiva del proyecto de explotación (Figura 3) no excedieron los Estándares de Calidad Ambiental (ECA) para Aire de $100 \mu \mathrm{g} / \mathrm{m}^{3}$ establecidos en el Decreto Supremo N 003-2017-MINAM (2017)

La concentración de $\mathrm{PM}_{2.5}$ (partículas menores a $2.5 \mu \mathrm{m}$ ) obtenido en el área efectiva del proyecto de explotación (Figura 4) no excedieron los Estándares de Calidad Ambiental (ECA) para Aire de $50 \mu \mathrm{g} / \mathrm{m}^{3}$ establecidos en el Decreto Supremo N $^{\circ}$ 003-2017-MINAM (2017)

Las emisiones de material particulado $\left(\mathrm{PM}_{10}\right.$ y $\left.\mathrm{PM}_{2.5}\right)$ que generan las labores del proyecto de explotación Cantera Camucha, no representan un riego significativo para la salud de los trabajadores ni para el medio ambiente.

Las medidas de mitigación y control ambiental implementadas por CMI Vilca Hermanos S.A.C. reducen eficientemente la generación de material particulado en el área efectiva del proyecto de explotación, por lo que se debe mantener y actualizar continuamente el Plan de Manejo Ambiental del proyecto.

\section{REFERENCIAS}

Blanco, E., \& Paricahua, H. (2020). Identificación y Valoración de Impactos Ambientales Generados por las Actividades de la Minería Informal, en el Cerro Luicho del Distrito de Colta, Provincia de Paucar del Sara Sara, Ayacucho" Erick [Universidad Tecnologica del Perú]. In Universidad Tecnologica del Perú. https://repositorio.utp.edu.pe/ handle/20.500.12867/3017

Blanco Obregón, J. M. (2018). El Ceibo (Ceiba sp.) como indicador de fuentes de contaminación atmosférica, en el Distrito de Chaclacayo, Lima, Perú. Revista Del Instituto De Investigación De La Facultad De Minas, Metalurgia Y Ciencias Geográficas, 20(39), 93-100. https:// revistasinvestigacion.unmsm.edu.pe/index.php/iigeo/ article/view/14171

Díaz Lazo, J. H. (2009). Contexto de la responsabilidad social minera y la gobernabilidad. Revista Del Instituto de Investigación de La Facultad de Ingeniería Geológica, Minera, Metalurgica y Geográfica, 12(23), 59-66. https:// revistasinvestigacion.unmsm.edu.pe/index.php/iigeo/ article/view/16684

Guzman Vasquez, J. J. Y. (2019). Evaluacion de la Calidad de Aire de la Central Termica de Ventanilla [Universidad Nacional Federico Villareal]. In Universiidad Nacional Federico Villareal. http://repositorio.unfv.edu.pe/handle/UNFV/4121

IDEAM. (2019). Informe del estado de la Calidad del Aire en Colombia 2018. Instituto de Hidrología, Meteorología y Estudios Ambientales, 61. http://www.ideam.gov.co/

La Rotta Latorre, Á. M., \& Torres Tovar, M. H. (2017). Explotación minera y sus impactos ambientales y en salud. El caso de Potosí en Bogotá. Saúde Em Debate, 41(112), 77-91. https://doi.org/10.1590/0103-1104201711207

MINAM. (2017). Decreto Supremo No 003-2017-MINAM .Aprueban Estándares de Calidad Ambiental (ECA) para Aire y establecen Disposiciones Complementarias. In El Peruano. https://cdn.www.gob.pe/uploads/document/ file/12871/DS-003-2017-MINAM.pdf

OMS. (2016). Contaminación atmosférica ambiental: una evaluación global de la exposición y la carga de morbilidad. https://www.who.int/phe/publications/air-pollution-globalassessment/en/ 
SINIA. (2019). Decreto Supremo No 010-2019-MINAM .- Decreto Supremo que aprueba el Protocolo Nacional de Monitoreo de la Calidad Ambiental del Aire. Decreto Supremo ${ }^{\circ}$ 010-2019-MINAM .- Decreto Supremo Que Aprueba El Protocolo Nacional de Monitoreo de La Calidad Ambiental Del Aire. www.peru.gob.pe
Valverde Flores, J. W. (2017). Evaluación de la calidad de aire en la intersección de la Av. Universitaria con Panamericana Norte - Los Olivos, Lima. Revista Del Instituto de Investigación de La Facultad de Ingeniería Geológica, Minera, Metalurgica y Geográfica, 19(38), 121-124. https://doi.org/10.15381/ iigeo.v19i38.13577 